\title{
Slow In and Slow Out Cartoon Animation Filter
}

\author{
David White, Kevin Loken, and Michiel van de Panne*
}

The University of British Columbia

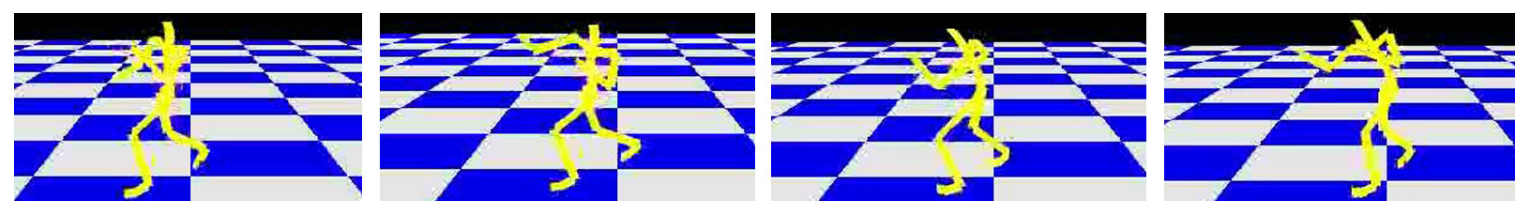

Figure 1: A motion capture example of boxing after filtering.

\begin{abstract}
"When using motion capture techniques for cartoonstyle animated characters it is essential to remind performers to do slow-ins and slow-outs." - Isaac Kerlow
\end{abstract}

\section{Introduction}

Motion capture motion is realistic, but is often dull and uninspiring when compared with hand-drawn animations. We present a filter that automatically adds slow in and slow out effects to motion capture motions to achieve more stylized, spirited motion. Our work is in part inspired by the "Cartoon Animation Filter" [Wang et al. 2006], which is proposes a filter for adding anticipation and followthrough effects.

Our filter is built upon the animation principle of slow in and slow out (SISO) [Thomas and Johnston 1981]. Slow in and slow out alters the timing of the animation so that the action is slowed down around important frames. Thomas and Johnston point out that overuse of this phenomenon will result in "a mechanical feel to the action", but proper usage will yield "a very spirited result".

\section{Our Filter}

Our SISO filter concentrates the motion of a given animated sequence around important (key) frames for a stylized effect. It can be used in two modes, one which preserves the original motion duration by speeding up the timing of in-between frames, and a second mode which preserves the timing of in-between motions, thereby resulting in a stylized clip of longer duration. We focus on applications to motion capture, but point out that our approach, modulo the keyframe selection, is as generally applicable as the aforementioned "Cartoon Animation Filter."

The first phase of our system takes arbitrary motion capture data and automatically identifies potential keyframes. This is done using Kinematic Centroid Segmentation (KCS) [Jenkins and Matarich 2004]. This process treats the centroid of each limb as a pendulum and automatically identifies the extreme points of the motion. KCS is performed independently on each of the four limbs and proximal segmentation boundaries are merged. These points of interest could be further filtered by additional criteria, or through user selection.

The second phase then builds a time-warp function [Witkin and Popovic 1995] that implements the desired SISO effect through a playback slow-down in the immediate vicinity of the identified keyframes. Animation play-back samples the time-warp function

\footnotetext{
*e-mail: $\{$ diwhite,kloken,van $\} @$ cs.ubc.ca
}

at equal frame time intervals along the $\mathrm{x}$-axis to compute the appropriate sample times $t^{\prime}$ for the original motion. Linear interpolation is used to reconstruct the pose at $t^{\prime}$ from temporally adjacent samples.

An example slow in and slow out warping function $g(\cdot)$ is illustrated in Figure 2, though any smooth monotonically increasing function that is bounded by the time of the original motion will suffice. The key is to have $\nabla g(\cdot) \rightarrow 0$ at each of the identified points of interest in the motion.

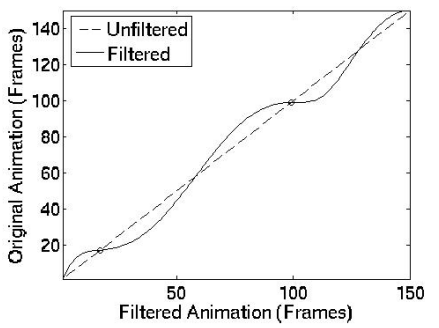

Figure 2: A time-warp function created to fit important motion at frames 17 and 99.

\section{Results}

We demonstrate the SISO filter to a martial arts kick, a soccer kick, and boxing. Sample frames from the boxing scenario are shown in Figure 1. The net effect of the SISO filter is to automatically slow the motion near keyframes and speed it up in less interesting areas.

The data used in this project was obtained from mocap.cs.cmu.edu. The database was created with funding from NSF EIA-0196217.

\section{References}

Jenkins, O. C., And Matarich, M. J. 2004. Performancederived behavior vocabularies: Data-driven acqusition of skills from motion. In International Journal of Humanoid Robotics.

Thomas, F., And Johnston, O. 1981. The Illusion of Life. Disney Editions, pp. 62.

Wang, J., Drucker, S. M., Agrawala, M., And Cohen, M. F. 2006. Cartoon animation filter. In SIGGRAPH 2006. 
Witkin, A., AND Popovic, Z. 1995. Motion warping. In SIGGRAPH 1995. 